Bull. Korean Math. Soc. 52 (2015), No. 3, pp. 1035-1046

http://dx.doi.org/10.4134/BKMS.2015.52.3.1035

\title{
HARDY SPACE OF LOMMEL FUNCTIONS
}

\author{
NiHAT YAĞMUR
}

ABSTRACT. In this work we present some geometric properties (like starlikeness and convexity of order $\alpha$ and also close-to-convexity of order $(1+\alpha) / 2)$ for normalized of Lommel functions of the first kind. In order to prove our main results, we use the technique of differential subordinations and some inequalities. Furthermore, we present some applications of convexity involving Lommel functions associated with the Hardy space of analytic functions, i.e., we obtain conditions for the function $h_{\mu, v}(z)$ to belong to the Hardy space $\mathcal{H}^{p}$.

\section{Introduction}

Let $\mathcal{H}$ be the set of all analytic functions on the open unit disk $\mathcal{U}=$ $\{z:|z|<1\}$. $\mathcal{H}^{\infty}$ denotes the space of all bounded functions on $\mathcal{H}$. $\mathcal{H}^{\infty}$ is a Banach algebra with respect to the norm $\|f\|_{\infty}=\sup \{|f(z)|: z \in \mathcal{U}\}$. For $p \in(0, \infty)$, we denote by $\mathcal{H}^{p}$ the space of all functions $f \in \mathcal{H}$ such that $|f|^{p}$ admits a harmonic majorant. $\mathcal{H}^{p}$ is a Banach space if we define the norm of $f$ to be the $p$-th root of the least harmonic majorant of $|f|^{p}$, taken in some fixed point $z \in \mathcal{U}$. For the function $f \in \mathcal{H}$, set

$$
M_{p}(r, f)=\left\{\begin{array}{cl}
\left(\frac{1}{2 \pi} \int_{0}^{2 \pi}\left|f\left(r e^{i \theta}\right)\right|^{p} d \theta\right)^{1 / p}, & p \in(0, \infty) \\
\max \{|f(z)|:|z| \leq r\}, & p=\infty
\end{array}\right.
$$

If $M_{p}(r, f)$ is bounded for all $r \in[0,1)$, then the function $f$ is said to belong to Hardy space $\mathcal{H}^{p}$. We note that for $0<p \leq s \leq \infty$, it can be shown that $\mathcal{H}^{s}$ is a subset of $\mathcal{H}^{p}$ (see the book of P. L. Duren [7]). Two known results about the Hardy spaces $\mathcal{H}^{p}$ are the followings [7]:

$$
\Re\left[f^{\prime}(z)\right]>0 \Longrightarrow \begin{cases}f^{\prime} \in \mathcal{H}^{q}, & q<1 \\ f \in \mathcal{H}^{q /(1-q)}, & q \in(0,1) .\end{cases}
$$

The study of Hardy spaces of special functions was studied first in [12]. This paper is motivated by [1] and [12].

Received July 21, 2014

2010 Mathematics Subject Classification. Primary 30C45; Secondary 33C10.

Key words and phrases. analytic function, Lommel functions, Hardy space. 
Let $\mathcal{A}$ denote the class of functions $f$ normalized by

$$
f(z)=z+\sum_{n=2}^{\infty} a_{n} z^{n}
$$

which are analytic in the open unit disk $\mathcal{U}$. Let $\mathcal{S}$ denote the subclass of $\mathcal{A}$ which are univalent in $\mathcal{U}$. Also let $\mathcal{S}^{*}(\alpha)$ and $\mathcal{C}(\alpha)$ denote the subclasses of $\mathcal{A}$ consisting of functions which are, respectively, starlike and convex of order $\alpha$ in $\mathcal{U}(0 \leq \alpha<1)$. Thus we have,

$$
\begin{gathered}
\mathcal{S}^{*}(\alpha)=\left\{f: f \in \mathcal{A} \text { and } \Re\left(\frac{z f^{\prime}(z)}{f(z)}\right)>\alpha,(z \in \mathcal{U} ; 0 \leq \alpha<1)\right\}, \\
\mathcal{C}(\alpha)=\left\{f: f \in \mathcal{A} \text { and } \Re\left(1+\frac{z f^{\prime \prime}(z)}{f^{\prime}(z)}\right)>\alpha,(z \in \mathcal{U} ; 0 \leq \alpha<1)\right\},
\end{gathered}
$$

and

$$
\mathcal{K}(\alpha)=\left\{f: f \in \mathcal{A} \text { and } \Re\left(\frac{f^{\prime}(z)}{g^{\prime}(z)}\right)>\alpha,(z \in \mathcal{U} ; 0 \leq \alpha<1 ; g \in \mathcal{C})\right\},
$$

where, for convenience,

$$
\mathcal{S}^{*}(0)=\mathcal{S}^{*}, \quad \mathcal{C}(0)=\mathcal{C}, \text { and } \mathcal{K}(0)=\mathcal{K} .
$$

For $\alpha<1$, Baricz (see [1]) introduced the class

$$
\mathcal{P}(\alpha)=\left\{p \in \mathcal{H}: \exists \eta \in \mathbb{R} \text { s.t. } p(0)=1, \Re\left[e^{i \eta}(p(z)-\alpha)\right]>0, z \in \mathcal{U}\right\}
$$

and $\mathcal{R}(\alpha)=\left\{f \in \mathcal{A}: f^{\prime} \in \mathcal{P}(\alpha)\right\}$, i.e.,

$$
\mathcal{R}(\alpha)=\left\{f \in \mathcal{A}: \exists \eta \in \mathbb{R} \text { s.t. } \Re\left[e^{i \eta}\left(f^{\prime}(z)-\alpha\right)\right]>0, z \in \mathcal{U}\right\} .
$$

When $\eta=0$, we denote $\mathcal{P}(\alpha)$ and $\mathcal{R}(\alpha)$ simply by $\mathcal{P}_{0}(\alpha)$ and $\mathcal{R}_{0}(\alpha)$, respectively; for $\alpha=0$ we denote $\mathcal{P}_{0}(\alpha)$ and $\mathcal{R}_{0}(\alpha)$ simply by $\mathcal{P}$ and $\mathcal{R}$, respectively. Finally, for functions $f, g \in \mathcal{A}$, given by $f(z)=z+\sum_{n=2}^{\infty} a_{n} z^{n}$ and $g(z)=z+\sum_{n=2}^{\infty} b_{n} z^{n}$, we define the Hadamard product (or convolution) of $f(z)$ and $g(z)$ by

$$
(f * g)(z):=z+\sum_{n=2}^{\infty} a_{n} b_{n} z^{n}=:(g * f)(z) \quad(z \in \mathcal{U}) .
$$

Lemma $1.1([9])$. Let $\mathbb{E}$ be a set in the complex plane $\mathbb{C}$ and $\psi: \mathbb{C}^{3} \times \mathcal{U} \rightarrow \mathbb{C}$ a function, that satisfies the admissibility condition $\psi(\rho i, \sigma, a+b i ; z) \notin \mathbb{E}$, where $z \in \mathcal{U}, \rho, \sigma, a, b \in \mathbb{R}$ with $a+\sigma \leq 0$ and $\sigma \leq-\left(1+\rho^{2}\right) / 2$. If $h$ : $\mathcal{U} \rightarrow \mathbb{C}$, which satisfies $h(0)=1$, is analytic and for all $z \in \mathcal{U}$ we have $\psi\left(h(z), z h^{\prime}(z), z^{2} h^{\prime \prime}(z) ; z\right) \in \mathbb{E}$, then $\Re\{h(z)\}>0$ for all $z \in \mathcal{U}$. In particular, if we only have $\psi: \mathbb{C}^{2} \times \mathcal{U} \rightarrow \mathbb{C}$, the admissibility condition reduces to $\psi(\rho i, \sigma ; z) \notin \mathbb{E}$ for all $z \in \mathcal{U}$ and $\rho, \sigma \in \mathbb{R}$ with $\sigma \leq-\left(1+\rho^{2}\right) / 2$. 
Lemma 1.2 ([11]). If $f \in \mathcal{A}$ satisfies the inequality

$$
\left|z f^{\prime \prime}(z)\right|<\frac{1-\alpha}{4} \quad(z \in \mathcal{U} ; 0 \leq \alpha<1)
$$

then

$$
\Re\left\{f^{\prime}(z)\right\}>\frac{1+\alpha}{4} \quad(z \in \mathcal{U} ; 0 \leq \alpha<1) .
$$

Lemma $1.3([14]) . \mathcal{P}_{0}(\alpha) * \mathcal{P}_{0}(\beta) \subset \mathcal{P}_{0}(\gamma)$, where $\gamma=1-2(1-\alpha)(1-\beta)$ and $\alpha, \beta<1$. The value of $\gamma$ is the best possible.

Lemma 1.4 ([13]). For $\alpha, \beta<1$ and $\gamma=1-2(1-\alpha)(1-\beta)$, we have $\mathcal{R}(\alpha) * \mathcal{R}_{0}(\beta) \subset \mathcal{R}(\gamma)$, or equivalently $\mathcal{P}(\alpha) * \mathcal{P}_{0}(\beta) \subset \mathcal{P}_{0}(\gamma)$.

Lemma $1.5([8])$. If the function $f$, convex of order $\alpha$, where $\alpha \in[0,1)$, is not of the form

$$
f(z)=\left\{\begin{array}{cc}
k+d z\left(1-z e^{i \gamma}\right)^{2 \alpha-1}, & \alpha \neq 1 / 2 \\
k+d \log \left(1-z e^{i \gamma}\right), & \alpha=1 / 2
\end{array}\right.
$$

for some complex numbers $k$ and $d$, and for some real number $\gamma$, then the following statements hold:

(i) There exists $\delta=\delta(f)>0$ s.t. $f^{\prime} \in \mathcal{H}^{\delta+1 /[2(1-\alpha)]}$;

(ii) If $\alpha \in[0,1 / 2)$, then there exists $\tau=\tau(f)>0$ s.t. $f \in \mathcal{H}^{\tau+1 /(1-2 \alpha)}$;

(iii) If $\alpha \geq 1 / 2$, then $f \in \mathcal{H}^{\infty}$.

\section{Starlikeness, convexity and close-to-convexity of the normalized Lommel functions}

Now, consider the Lommel function of the first kind $s_{\mu, v}$ is a particular solution of the inhomogeneous Bessel differential equation (see, for details, [15] and [4]):

$$
z^{2} w^{\prime \prime}(z)+z w^{\prime}(z)+\left(z^{2}-v^{2}\right) w(z)=z^{\mu+1}
$$

and it can be expressed in terms of a hypergeometric series

$$
s_{\mu, v}(z)=\frac{z^{\mu+1}}{(\mu-v+1)(\mu+v+1)}{ }_{1} F_{2}\left(1 ; \frac{\mu-v+3}{2}, \frac{\mu+v+3}{2} ;-\frac{z^{2}}{4}\right),
$$

where $\mu \pm v$ is not negative odd integer. Observe that the Lommel function $s_{\mu, v}$ does not belong to class $\mathcal{A}$. Thus, it is natural to consider the following normalization of the Lommel function of the first kind

$$
\begin{aligned}
h_{\mu, v}(z) & =(\mu-v+1)(\mu+v+1) z^{\frac{1-\mu}{2}} s_{\mu, v}(\sqrt{z}) \\
& =z+\sum_{n=1}^{\infty} \frac{(-1 / 4)^{n}}{(K)_{n}(F)_{n}} z^{n+1},
\end{aligned}
$$

where $K=\frac{\mu-v+3}{2}, F=\frac{\mu+v+3}{2}$ and $(\lambda)_{n}$ shows Pochhammer (or Appell) symbol which defined in terms of Euler's gamma functions such that $(\lambda)_{n}=$ $\Gamma(\lambda+n) / \Gamma(\lambda)=\lambda(\lambda+1) \cdots(\lambda+n-1)$. Clearly the function $h_{\mu, v}(z)$ belongs to the class $\mathcal{A}$. 
It is important to mention related to geometric properties of special functions here that Baricz and Ponnusamy (see [2], [3] and [5]) obtained geometric properties of generalized Bessel functions, recently Yağmur and Orhan [10, 16] obtained geometric properties of generalized Struve functions. Furthermore, very recently Baricz and Szász [6] considered the starlikeness and close-toconvexity of the derivatives of a normalized form of $s_{\mu-\frac{1}{2}, \frac{1}{2}}$. In this section, we get some geometric properties of the function $h_{\mu, v}(z)$ which normalized Lommel functions of the first kind $s_{\mu, v}(z)$.

Now, we have the following theorem for starlikeness and convexity of order $\alpha$ and also close-to-convexity of order $\frac{1+\alpha}{2}$ for the function $h_{\mu, v}(z)$.

Theorem 2.1. Let $\alpha \in[0,1), \mu, v \in \mathbb{R}$ where $\mu \pm v$ is not negative odd integer, $M=4(K+1)(F+1)=(\mu+5)^{2}-v^{2}$ and $N=4 K F=(\mu+3)^{2}-v^{2}$. Then for all $z \in \mathcal{U}$ the following assertions are true:

(i) If $\mu>-5+\sqrt{2+v^{2}}$ and $\frac{M(M-1)}{(M-2)(M N-M-N)}<1-\alpha$, then $h_{\mu, v}(z) \in$ $\mathcal{S}^{*}(\alpha)$

(ii) If $\mu>-5+\sqrt{3+v^{2}}$ and $\frac{2 M(2 M-3)}{(M-3)(2 M N-4 M-3 N)}<1-\alpha$, then $h_{\mu, v}(z) \in$ $\mathcal{C}(\alpha)$

(iii) If $\mu>-5+\sqrt{3+v^{2}}$ and $\frac{8 M}{(M-3) N}<1-\alpha$, then the function $h_{\mu, v}(z) \in$ $\mathcal{K}\left(\frac{1+\alpha}{2}\right)$ and so $\Re\left\{h_{\mu, v}^{\prime}(z)\right\}>\frac{1+\alpha}{2}$.

Proof. (i) We use the inequality $\left|\frac{z h_{\mu, v}^{\prime}(z)}{h_{\mu, v}(z)}-1\right|<1-\alpha$ to prove the starlikeness of order $\alpha$ for the function $h_{\mu, v}(z)$. So, by using the well-known triangle inequality

the inequalities

$$
\left|z_{1}+z_{2}\right| \leq\left|z_{1}\right|+\left|z_{2}\right|
$$

$$
\begin{aligned}
& 2^{n-1}(K+1)_{n-1}(F+1)_{n-1} \geq n(K+1)^{n-1}(F+1)^{n-1} \\
& (n \in \mathbb{N}, K+1>0, F+1>0),
\end{aligned}
$$

and the identity $(\lambda)_{n}=\lambda(\lambda+1)_{n-1}$, we have

$$
\begin{aligned}
\left|h_{\mu, v}^{\prime}(z)-\frac{h_{\mu, v}(z)}{z}\right| & =\left|\sum_{n=1}^{\infty} \frac{n(-1 / 4)^{n}}{(K)_{n}(F)_{n}} z^{n}\right| \\
& \leq \frac{1}{4 K F} \sum_{n=1}^{\infty} \frac{n}{4^{n-1}(K+1)_{n-1}(F+1)_{n-1}} \\
& \leq \frac{1}{4 K F} \sum_{n=1}^{\infty}\left(\frac{1}{2(K+1)(F+1)}\right)^{n-1} \\
& =\frac{1}{N} \sum_{n=1}^{\infty}\left(\frac{2}{M}\right)^{n-1} \\
& =\frac{M}{N(M-2)}, \quad(M>2) .
\end{aligned}
$$


Furthermore, if we use reverse triangle inequality

$$
\left|z_{1}-z_{2}\right| \geq|| z_{1}|-| z_{2}||
$$

and the inequality $(K+1)_{n-1}(F+1)_{n-1} \geq(K+1)^{n-1}(F+1)^{n-1}$, then we get

$$
\begin{aligned}
\left|\frac{h_{\mu, v}(z)}{z}\right| & =\left|1+\sum_{n=1}^{\infty} \frac{(-1 / 4)^{n}}{(K)_{n}(F)_{n}} z^{n}\right| \\
& \geq 1-\frac{1}{4 K F} \sum_{n=1}^{\infty} \frac{1}{4^{n-1}(K+1)_{n-1}(F+1)_{n-1}} \\
& \geq 1-\frac{1}{4 K F} \sum_{n=1}^{\infty}\left(\frac{1}{4(K+1)(F+1)}\right)^{n-1} \\
& =1-\frac{1}{N} \sum_{n=1}^{\infty}\left(\frac{1}{M}\right)^{n-1} \\
& =\frac{N(M-1)-M}{N(M-1)}(M>2) .
\end{aligned}
$$

Next, by combining the inequalities (2.4) with (2.5), we immediately see that

$$
\left|\frac{z h_{\mu, v}^{\prime}(z)}{h_{\mu, v}(z)}-1\right| \leq \frac{M(M-1)}{(M-2)(M N-M-N)} .
$$

So, for $0 \leq \alpha<1-\frac{M(M-1)}{(M-2)(M N-M-N)}$, we obtain

$$
\left|\frac{z h_{\mu, v}^{\prime}(z)}{h_{\mu, v}(z)}-1\right|<\frac{M(M-1)}{(M-2)(M N-M-N)}<1-\alpha .
$$

The inequalities $K+1>0, F+1>0$ and $M>2$ satisfy that $\mu>-5+\sqrt{2+v^{2}}$. So the first part of the theorem is complete.

(ii) To show the convexity of order $\alpha$ of the function $h_{\mu, v}(z)$, we show the inequality $\left|\frac{z h_{\mu, v}^{\prime \prime}(z)}{h_{\mu, v}(z)}\right|<1-\alpha$ satisfies for appropriate values of the parameters $\mu, v$ and $\alpha$. By using the triangle inequality and the inequalities

$$
\begin{aligned}
& 3^{n-1} \geq \frac{n(n+1)}{2}, \quad(K+1)_{n-1}(F+1)_{n-1} \geq(K+1)^{n-1}(F+1)^{n-1} \\
& (n \in \mathbb{N}, K+1>0, F+1>0)
\end{aligned}
$$

we arrive at the following

$$
\begin{aligned}
\left|z h_{\mu, v}^{\prime \prime}(z)\right| & =\left|\sum_{n=1}^{\infty} \frac{n(n+1)(-1 / 4)^{n}}{(K)_{n}(F)_{n}} z^{n}\right| \\
& \leq \frac{1}{2 K F} \sum_{n=1}^{\infty} \frac{n(n+1) / 2}{4^{n-1}(K+1)_{n-1}(F+1)_{n-1}}
\end{aligned}
$$




$$
\begin{aligned}
& =\frac{1}{2 K F} \sum_{n=1}^{\infty} \frac{n(n+1) / 2}{3^{n-1}(4 / 3)^{n-1}(K+1)_{n-1}(F+1)_{n-1}} \\
& \leq \frac{1}{2 K F} \sum_{n=1}^{\infty}\left[\frac{3}{4(K+1)(F+1)}\right]^{n-1} \\
& =\frac{2}{N} \sum_{n=1}^{\infty}\left[\frac{3}{M}\right]^{n-1} \\
& =\frac{2 M}{N(M-3)}(M>3) .
\end{aligned}
$$

Moreover, if we use reverse triangle inequality and the inequalities

$$
\begin{aligned}
& (3 / 2)^{n-1} \geq \frac{n+1}{2}, \quad(K+1)_{n-1}(F+1)_{n-1} \geq(K+1)^{n-1}(F+1)^{n-1} \\
& (n \in \mathbb{N}, K+1>0, F+1>0),
\end{aligned}
$$

then we have

$$
\begin{aligned}
\left|h_{\mu, v}^{\prime}(z)\right| & =\left|1+\sum_{n=1}^{\infty} \frac{(n+1)(-1 / 4)^{n}}{(K)_{n}(F)_{n}} z^{n}\right| \\
& \geq 1-\sum_{n=1}^{\infty} \frac{(n+1)}{4^{n}(K)_{n}(F)_{n}} \\
& =1-\frac{1}{2 K F} \sum_{n=1}^{\infty} \frac{(n+1) / 2}{4^{n-1}(K+1)_{n-1}(F+1)_{n-1}} \\
& \geq 1-\frac{1}{2 K F} \sum_{n=1}^{\infty} \frac{(n+1) / 2}{(3 / 2)^{n-1}(8 / 3)^{n-1}(K+1)_{n-1}(F+1)_{n-1}} \\
& \geq 1-\frac{1}{2 K F} \sum_{n=1}^{\infty}\left[\frac{3}{8(K+1)(F+1)}\right]^{n-1} \\
& =1-\frac{2}{N} \sum_{n=1}^{\infty}\left[\frac{3}{2 M}\right]^{n-1} \\
& =\frac{2 M N-4 M-3 N}{N(2 M-3)}(M>3 / 2) .
\end{aligned}
$$

Next, by combining the inequalities (2.6) with (2.7), we immediately deduce that

$$
\left|\frac{z h_{\mu, v}^{\prime \prime}(z)}{h_{\mu, v}^{\prime}(z)}\right| \leq \frac{2 M(2 M-3)}{(M-3)(2 M N-4 M-3 N)}<1-\alpha
$$

if $0 \leq \alpha<1-\frac{2 M(2 M-3)}{(M-3)(2 M N-4 M-3 N)}$. The inequalities $K+1>0, F+1>0$ and $M>3$ hold if and only if $\mu>-5+\sqrt{3+v^{2}}$. 
(iii) By using the inequality (2.6) and Lemma 1.2 we obtain that

$$
\left|z h_{\mu, v}^{\prime \prime}(z)\right| \leq \frac{2 M}{N(M-3)}<\frac{1-\alpha}{4}
$$

for $0 \leq \alpha<1-\frac{8 M}{(M-3) N}$ and $\mu>-5+\sqrt{3+v^{2}}$. This implies that $h_{\mu, v}(z) \in$ $\mathcal{K}\left(\frac{1+\alpha}{2}\right)$ and so $\Re\left\{h_{\mu, v}^{\prime}(z)\right\}>\frac{1+\alpha}{2}$.

The proof is complete.

By taking $\alpha=0$ in Theorem 2.1 we get the following corollary.

Corollary 2.2. If $\mu, v \in \mathbb{R}$ where $\mu \pm v$ is not negative odd integer, $M=$ $4(K+1)(F+1)=(\mu+5)^{2}-v^{2}$ and $N=4 K F=(\mu+3)^{2}-v^{2}$, then for all $z \in \mathcal{U}$ the following assertions are valid:

(i) If $\mu>-5+\sqrt{2+v^{2}}$ and $\frac{M(M-1)}{(M-2)(M N-M-N)}<1$, then $h_{\mu, v}(z) \in \mathcal{S}^{*}$;

(ii) If $\mu>-5+\sqrt{3+v^{2}}$ and $\frac{2 M(2 M-3)}{(M-3)(2 M N-4 M-3 N)}<1$, then $h_{\mu, v}(z) \in \mathcal{C}$;

(iii) If $\mu>-5+\sqrt{3+v^{2}}$ and $\frac{8 M}{(M-3) N}<1$, then the function $h_{\mu, v}(z) \in$ $\mathcal{K}(1 / 2)$ and so $\Re\left\{h_{\mu, v}^{\prime}(z)\right\}>1 / 2$.

Theorem 2.3. Let $\mu>-1, v \in \mathbb{R}$ where $\mu \pm v$ is not negative odd integer, and $\alpha \in[0,1)$ the function $\frac{h_{\mu, v}(z)}{z}$ satisfy the following property:

If

$$
\frac{8(\mu+1)\left[(\mu+1)(\mu+3)-v^{2}\right]-1}{16(\mu+1)^{2}} \geq \frac{\alpha^{2}}{(1-\alpha)^{2}},
$$

then $\frac{h_{\mu, v}}{z} \in \mathcal{P}_{0}(\alpha)$ for all $z \in \mathcal{U}$.

Proof. Define the function $g: \mathcal{U} \rightarrow \mathbb{C}$ by

$$
g(z)=\frac{h_{\mu, v}(z) / z-\alpha}{1-\alpha} .
$$

Since $s_{\mu, v}(z)$ satisfies $(2.1), g$ will satisfy the following differential equation:

$$
\begin{aligned}
& z^{2} g^{\prime \prime}(z)+(\mu+2) z g^{\prime}(z)+\left(\frac{z}{4}+\frac{(\mu+1)^{2}-v^{2}}{2}\right)\left(g(z)+\frac{\alpha}{1-\alpha}\right) \\
& -\left(\frac{(\mu+1)^{2}-v^{2}}{2(1-\alpha)}\right)=0 .
\end{aligned}
$$

If we use $\psi(r, s, t ; z)=t+(\mu+2) s+\left(\frac{z}{4}+\frac{(\mu+1)^{2}-v^{2}}{2}\right)\left(r+\frac{\alpha}{1-\alpha}\right)-\left(\frac{(\mu+1)^{2}-v^{2}}{2(1-\alpha)}\right)$ and $\mathbb{E}=\{0\}$, we see that equation (2.8) implies $\psi\left(g(z), z g^{\prime}(z), z^{2} g^{\prime \prime}(z) ; z\right) \in \mathbb{E}$ for all $z \in \mathcal{U}$. Now we use Lemma 1.1 to prove that $\Re\{g(z)\}>0$ for all $z \in \mathcal{U}$. If we put $z=x+i y$, where $x, y \in \mathbb{R}$, then

$$
\begin{aligned}
& \Re\{\psi(\rho i, \sigma, a+b i ; x+i y)\} \\
= & (a+\sigma)+(\mu+1) \sigma+\frac{y \rho}{4}+\left(\frac{x}{4}+\frac{(\mu+1)^{2}-v^{2}}{2}\right) \frac{\alpha}{1-\alpha}-\left(\frac{(\mu+1)^{2}-v^{2}}{2(1-\alpha)}\right)
\end{aligned}
$$


for all $\rho, \sigma, a, b \in \mathbb{R}$. Let $\rho, \sigma, a, b \in \mathbb{R}$ satisfy $a+\sigma \leq 0$ and $\sigma \leq-\left(1+\rho^{2}\right) / 2$. Since $\mu>-1$, we have

$$
\begin{aligned}
& \Re\{\psi(\rho i, \sigma, a+b i ; x+i y)\} \\
\leq & -\frac{(\mu+1)}{2} \rho^{2}+\frac{y \rho}{4}-\frac{(\mu+1)}{2}+\left(\frac{x}{4}+\frac{(\mu+1)^{2}-v^{2}}{2}\right) \frac{\alpha}{1-\alpha} \\
& -\left(\frac{(\mu+1)^{2}-v^{2}}{2(1-\alpha)}\right) .
\end{aligned}
$$

Set $Q_{1}(\rho)=-\frac{(\mu+1)}{2} \rho^{2}+\frac{y \rho}{4}-\frac{(\mu+1)}{2}+\left(\frac{x}{4}+\frac{(\mu+1)^{2}-v^{2}}{2}\right) \frac{\alpha}{1-\alpha}-\left(\frac{(\mu+1)^{2}-v^{2}}{2(1-\alpha)}\right)$. This value will be strictly negative for all real $\rho$, because the discriminant $\Delta_{1}$ of $Q_{1}(\rho)$ satisfies

$$
\begin{aligned}
\Delta_{1}= & \frac{y^{2}}{16}+2(\mu+1)\left[-\frac{(\mu+1)}{2}+\left(\frac{x}{4}+\frac{(\mu+1)^{2}-v^{2}}{2}\right) \frac{\alpha}{1-\alpha}-\left(\frac{(\mu+1)^{2}-v^{2}}{2(1-\alpha)}\right)\right] \\
< & \frac{1}{16}\left(1-x^{2}\right)-(\mu+1)^{2} \\
& +2(\mu+1)\left[\left(\frac{x}{4}+\frac{(\mu+1)^{2}-v^{2}}{2}\right) \frac{\alpha}{1-\alpha}-\left(\frac{(\mu+1)^{2}-v^{2}}{2(1-\alpha)}\right)\right] \\
= & -\frac{1}{16} x^{2}+\frac{(\mu+1) \alpha}{2(1-\alpha)} x-\frac{(\mu+1)\left[(\mu+1)(\mu+3)-v^{2}\right]}{2}+\frac{1}{16} \\
= & : Q_{2}(x) \leq 0,
\end{aligned}
$$

whenever $x^{2}+y^{2}<1$ and the discriminant $\Delta_{2}$ of $Q_{2}(x)$ is negative. $\Delta_{2}$ has the form

$$
\Delta_{2}=\frac{(\mu+1)^{2} \alpha^{2}}{4(1-\alpha)^{2}}-\frac{(\mu+1)\left[(\mu+1)(\mu+3)-v^{2}\right]}{8}+\frac{1}{64}
$$

and this is negative if and only if we have $\frac{8(\mu+1)\left[(\mu+1)(\mu+3)-v^{2}\right]-1}{16(\mu+1)^{2}}>\frac{\alpha^{2}}{(1-\alpha)^{2}}$. Hence by Lemma 1.1 we conclude that

$$
\Re\{g(z)\}=\Re\left[\frac{1}{1-\alpha}\left(h_{\mu, v}(z) / z-\alpha\right)\right]>0 \text { for all } z \in \mathcal{U},
$$

and this implies that $\Re\left\{h_{\mu, v}(z) / z\right\}>\alpha$ for all $z \in \mathcal{U}$, as we required.

If we take $\alpha=0$ in Theorem 2.3 we get the following corollary.

Corollary 2.4. If $\mu>-1, v \in \mathbb{R}$ where $\mu \pm v$ is not negative odd integer, and

$$
(\mu+1)\left[(\mu+1)(\mu+3)-v^{2}\right] \geq 1 / 8,
$$

then $\frac{h_{\mu, v}}{z} \in \mathcal{P}$ for all $z \in \mathcal{U}$. 


\section{Hardy space of Lommel functions}

Recently, Ponnusamy [12] studied the Hardy space of hypergeometric functions. By using the same idea Baricz [1] obtained the conditions for generalized Bessel functions to belong to Hardy space, and similarly Yağmur and Orhan [17] studied the same problem for generalized Struve functions. Now, we get the conditions for normalized Lommel functions of the first kind to belong to Hardy space.

Theorem 3.1. Let $\alpha \in[0,1), \mu, v \in \mathbb{R}$ where $\mu \pm v$ is not negative odd integer, and $M=(\mu+5)^{2}-v^{2}, N=(\mu+3)^{2}-v^{2}$. If $\mu>-5+\sqrt{3+v^{2}}$ and $\frac{2 M(2 M-3)}{(M-3)(2 M N-4 M-3 N)}<1-\alpha$, then:

(i) for $\alpha \in[0,1 / 2)$, we have $h_{\mu, v} \in \mathcal{H}^{1 /(1-2 \alpha)}$;

(ii) for $\alpha \geq 1 / 2$, we have $h_{\mu, v} \in \mathcal{H}^{\infty}$.

Proof. By the definition of the Gaussian hypergeometric function

$$
{ }_{2} F_{1}(a, b, c ; z)=\sum_{n=0}^{\infty} \frac{(a)_{n}(b)_{n}}{(c)_{n}} \frac{z^{n}}{n !},
$$

we have

$$
\begin{aligned}
k+\frac{d z}{\left(1-z e^{i \gamma}\right)^{1-2 \alpha}} & =k+d z_{2} F_{1}\left(1,1-2 \alpha, 1 ; z e^{i \gamma}\right) \\
& =k+d \sum_{n=0}^{\infty} \frac{(1-2 \alpha)}{n !} e^{i \gamma n} z^{n+1}
\end{aligned}
$$

for $k, d \in \mathbb{C}, \alpha \neq 1 / 2$ and for real $\gamma$. On the other hand

$$
\begin{aligned}
k+d \log \left(1-z e^{i \gamma}\right) & =k-d z{ }_{2} F_{1}\left(1,1,2 ; z e^{i \gamma}\right) \\
& =k-d \sum_{n=0}^{\infty} \frac{1}{n+1} e^{i \gamma n} z^{n+1} .
\end{aligned}
$$

Therefore the function $h_{\mu, v}$ is not of the forms $k+d z\left(1-z e^{i \gamma}\right)^{2 \alpha-1}($ for $\alpha \neq 1 / 2)$ and $k+d \log \left(1-z e^{i \gamma}\right.$ ) (for $\alpha=1 / 2$ ). We know that by part (ii) of Theorem 2.1, $h_{\mu, v}$ is convex of order $\alpha$. Hence by Lemma 1.5, the proof is completed.

Theorem 3.2. Let $\mu>-1, v \in \mathbb{R}$ where $\mu \pm v$ is not negative odd integer. If

$$
(\mu+1)\left[(\mu+1)(\mu+3)-v^{2}\right] \geq 1 / 8
$$

and $f \in \mathcal{R}$, then the convolution $h_{\mu, v} * f$ is in $\mathcal{H}^{\infty} \cap \mathcal{R}$.

Proof. Define $u(z)=h_{\mu, v}(z) * f(z)$. Then $u^{\prime}(z)=\frac{h_{\mu, v}(z)}{z} * f^{\prime}(z)$. From Corollary 2.4 we have $\frac{h_{\mu, v}}{z} \in \mathcal{P}$. Since $f \in \mathcal{R}$, it follows from Lemma 1.3 that $u \in \mathcal{R}$. It is clear that $\frac{h_{\mu, v}(z)}{z}$ is an entire function and consequently the function $u$ is itself an entire function. Consequently the function $u$ is bounded, which completes the proof. 
Theorem 3.3. Let $\alpha \in[0,1), \mu>-1, v \in \mathbb{R}$ and $\mu \pm v$ is not negative odd integer and

$$
\frac{8(\mu+1)\left[(\mu+1)(\mu+3)-v^{2}\right]-1}{16(\mu+1)^{2}} \geq \frac{\alpha^{2}}{(1-\alpha)^{2}} .
$$

If we have $f \in \mathcal{R}(\beta)$, with $\beta<1$, then $h_{\mu, v} * f \in \mathcal{R}(\gamma)$ where $\gamma=1-2(1-$ $\beta)(1-\alpha)$.

Proof. Set $u(z)=h_{\mu, v}(z) * f(z)$. Then we get $u^{\prime}(z)=\frac{h_{\mu, v}(z)}{z} * f^{\prime}(z)$. From Theorem 2.3 we have $\frac{h_{\mu, v}}{z} \in \mathcal{P}_{0}(\alpha)$. By using Lemma 1.4 and the fact that $f^{\prime} \in \mathcal{P}(\beta)$, we immediately obtain that the function $u^{\prime}$ belongs to $\mathcal{P}(\gamma)$, where $\gamma=1-2(1-\beta)(1-\alpha)$. In fact, $u^{\prime} \in \mathcal{P}(\gamma)$ is equivalent to $u \in \mathcal{R}(\gamma)$, hence the proof is complete.

Corollary 3.4. Let $\alpha, \mu, v$ satisfy the hypotheses of Theorem 3.3. If $f \in \mathcal{R}(\beta)$, where $\beta=(1-2 \alpha) /(2-2 \alpha)$, then $h_{\mu, v} * f \in \mathcal{R}(0)$.

Taking $\alpha=0$ in the above corollary we obtain the next result.

Corollary 3.5. Let $\alpha, \mu, v$ satisfy the hypotheses of Theorem 3.2. If $f \in$ $\mathcal{R}(1 / 2)$, then $h_{\mu, v} * f \in \mathcal{R}(0)$.

\section{Particular cases}

We end this paper with particular cases of all the theorems and corollaries. Choosing special cases for $\mu$, and $v$ in (2.2) we obtain the following functions:

$$
\begin{aligned}
& h_{\frac{1}{2}, \frac{1}{2}}(z)=2(1-\cos \sqrt{z}), \quad h_{\frac{3}{2}, \frac{1}{2}}(z)=6\left(1-\frac{\sin \sqrt{z}}{\sqrt{z}}\right) \\
& h_{\frac{5}{2}, \frac{1}{2}}(z)=12\left(\frac{z+2 \cos \sqrt{z}-2}{z}\right) .
\end{aligned}
$$

By using Theorem 2.1, Theorem 2.3 and Theorem 3.3 we get the following three corollaries.

Corollary 4.1. (i) If $0 \leq \alpha<\alpha_{0}$ where $\alpha_{0} \simeq 0.90229 \cdots$, then $h_{\frac{1}{2}, \frac{1}{2}} \in \mathcal{S}^{*}(\alpha)$.

(ii) If $0 \leq \alpha<\alpha_{1}$ where $\alpha_{1} \simeq 0.77541 \cdots$, then $h_{\frac{1}{2}, \frac{1}{2}} \in \mathcal{C}(\alpha)$.

(iii) If $0 \leq \alpha<\alpha_{2}$ where $\alpha_{2} \simeq 0.25926 \cdots$, then $h_{\frac{1}{2}, \frac{1}{2}} \in \mathcal{K}\left(\frac{1+\alpha}{2}\right)$.

(iv) If $0 \leq \alpha<\alpha_{3}$ where $\alpha_{3} \simeq 0.64419 \cdots$, then $\frac{h_{\frac{1}{2}, \frac{1}{2}}}{z} \in \mathcal{P}_{0}(\alpha)$. If we have $f \in \mathcal{R}(\beta)$ with $\beta<1$, then $h_{\frac{1}{2}}, \frac{1}{2} * f \in \mathcal{R}(\gamma)$ where $\gamma=1-2(1-\beta)(1-\alpha)$. In particular, if $f \in \mathcal{R}(\beta)$ where $\beta=(1-2 \alpha) /(2-2 \alpha)$, then $h_{\frac{1}{2}, \frac{1}{2}} * f \in \mathcal{R}(0)$.

Corollary 4.2. (i) If $0 \leq \alpha<\alpha_{4}$ where $\alpha_{4} \simeq 0.94467 \cdots$, then $h_{\frac{3}{2}, \frac{1}{2}} \in \mathcal{S}^{*}(\alpha)$.

(ii) If $0 \leq \alpha<\alpha_{5}$ where $\alpha_{5} \simeq 0.87985 \cdots$, then $h_{\frac{3}{2}, \frac{1}{2}} \in \mathcal{C}(\alpha)$.

(iii) If $0 \leq \alpha<\alpha_{6}$ where $\alpha_{6} \simeq 0.56923 \cdots$, then $h_{\frac{3}{2}, \frac{1}{2}} \in \mathcal{K}\left(\frac{1+\alpha}{2}\right)$. 
(iv) If $0 \leq \alpha<\alpha_{7}$ where $\alpha_{7} \simeq 0.59675 \cdots$, then $\frac{h_{\frac{3}{2}, \frac{1}{2}}}{z} \in \mathcal{P}_{0}(\alpha)$. If we have $f \in \mathcal{R}(\beta)$ with $\beta<1$, then $h_{\frac{3}{2}}, \frac{1}{2} * f \in \mathcal{R}(\gamma)$ where $\gamma=1-2(1-\beta)(1-\alpha)$. In particular, if $f \in \mathcal{R}(\beta)$ where $\beta=(1-2 \alpha) /(2-2 \alpha)$, then $h_{\frac{3}{2}, \frac{1}{2}} * f \in \mathcal{R}(0)$.

Corollary 4.3. (i) If $0 \leq \alpha<\alpha_{8}$ where $\alpha_{8} \simeq 0.96421 \cdots$, then $h_{\frac{5}{2}, \frac{1}{2}} \in \mathcal{S}^{*}(\alpha)$.

(ii) If $0 \leq \alpha<\alpha_{9}$ where $\alpha_{9} \simeq 0.92438 \cdots$, then $h_{\frac{5}{2}, \frac{1}{2}} \in \mathcal{C}(\alpha)$.

(iii) If $0 \leq \alpha<\alpha_{10}$ where $\alpha_{10} \simeq 0.71824 \cdots$, then $h_{\frac{5}{2}, \frac{1}{2}} \in \mathcal{K}\left(\frac{1+\alpha}{2}\right)$.

(iv) If $0 \leq \alpha<\alpha_{11}$ where $\alpha_{11} \simeq 0.62207 \cdots$, then $\frac{h_{\frac{5}{2}, \frac{1}{2}}}{z} \in \mathcal{P}_{0}(\alpha)$. If we have $f \in \mathcal{R}(\beta)$ with $\beta<1$, then $h_{\frac{5}{2}, \frac{1}{2}} * f \in \mathcal{R}(\gamma)$ where $\gamma={ }_{1}^{z}-2(1-\beta)(1-\alpha)$. In particular, if $f \in \mathcal{R}(\beta)$ where $\beta=(1-2 \alpha) /(2-2 \alpha)$, then $h_{\frac{5}{2}, \frac{1}{2}} * f \in \mathcal{R}(0)$.

By using Theorem 3.1 we get the following corollary.

Corollary 4.4. (i) For $\alpha \in[0,1 / 2)$, we have $h_{\frac{1}{2}, \frac{1}{2}} \in \mathcal{H}^{1 /(1-2 \alpha)}$, and if $\alpha \in$ $\left[1 / 2, \alpha_{1}\right)$ where $\alpha_{1} \simeq 0.77541 \cdots$, then $h_{\frac{1}{2}, \frac{1}{2}} \in \mathcal{H}^{\infty}$.

(ii) For $\alpha \in[0,1 / 2)$, we have $h_{\frac{3}{2}, \frac{1}{2}} \in \mathcal{H}^{1 /(1-2 \alpha)}$, and if $\alpha \in\left[1 / 2, \alpha_{5}\right)$ where $\alpha_{5} \simeq 0.87985 \cdots$, then $h_{\frac{3}{2}, \frac{1}{2}} \in \mathcal{H}^{\infty}$.

(iii) For $\alpha \in[0,1 / 2)$, we have $h_{\frac{5}{2}, \frac{1}{2}} \in \mathcal{H}^{1 /(1-2 \alpha)}$, and if $\alpha \in\left[1 / 2, \alpha_{9}\right)$ where $\alpha_{9} \simeq 0.92438 \cdots$, then $h_{\frac{5}{2}, \frac{1}{2}} \in \mathcal{H}^{\infty}$.

If we use Theorem 3.2 and Corollary 3.5, then we have the following corollary.

Corollary 4.5. If $f \in \mathcal{R}$, then the convolutions $h_{\frac{1}{2}, \frac{1}{2}} * f, h_{\frac{3}{2}, \frac{1}{2}} * f$ and $h_{\frac{5}{2}, \frac{1}{2}}$ $* f$ are in $\mathcal{H}^{\infty} \cap \mathcal{R}$. Moreover, if $f \in \mathcal{R}(1 / 2)$, then $h_{\frac{1}{2}, \frac{1}{2}} * f, h_{\frac{3}{2}, \frac{1}{2}} * f$ and $h_{\frac{5}{2}, \frac{1}{2}}$ *f are in $\mathcal{R}(0)$.

\section{References}

[1] Á. Baricz, Bessel transforms and Hardy space of generalized Bessel functions, Mathematica 48(71) (2006), no. 2, 127-136.

$[2] \_$, Geometric properties of generalized Bessel functions, Publ. Math. Debrecen $\mathbf{7 3}$ (2008), no. 1-2, 155-178.

[3] Generalized Bessel functions of the first kind, Lecture Notes in Mathematics, vol. 1994, Springer-Verlag, Berlin, 2010.

[4] Á. Baricz and S. Koumandos, Turan type inequalities for some lommel functions of the first kind, arxiv:1308.6477.

[5] Á. Baricz and S. Ponnusamy, Starlikeness and convexity of generalized Bessel functions, Integral Transforms Spec. Funct. 21 (2010), no. 9-10, 641-653.

[6] Á. Baricz and R. Szász, Close-to-convexity of some special functions and their derivatives, arxiv:1402.0692.

[7] P. L. Duren, Theory of $\mathcal{H}^{p}$ Spaces, Academic Press, New York/London, 1970.

[8] P. J. Eenigenburg and F. R. Keogh, The Hardy class of some univalent functions and their derivatives, Michigan Math. J. 17 (1970), 335-346.

[9] S. S. Miller and P. T. Mocanu, Differential subordinations and inequalities in the complex plane, J. Differential Equations 67 (1987), no. 2, 199-211.

[10] H. Orhan and N. Yağmur, Geometric properties of generalized struve functions, An. Ştiinţ. Univ. Al. I. Cuza Iaşi. Mat. (N.S.) (in press). 
[11] S. Owa, M. Nunokawa, H. Saitoh, and H. M. Srivastava, Close-to-convexity, starlikeness, and convexity of certain analytic functions, Appl. Math. Lett. 15 (2002), no. 1, 63-69.

[12] S. Ponnusamy, The Hardy space of hypergeometric functions, Complex Variables Theory Appl. 29 (1996), no. 1, 83-96.

[13] _ Inclusion theorems for convolution product of second order polylogarithms and functions with the derivative in a halfplane, Rocky Mountain J. Math. 28 (1998), no. $2,695-733$.

[14] J. Stankiewicz and Z. Stankiewicz, Some applications of the Hadamard convolutions in the theory of functions, Ann. Univ. Mariae Curie-Sklodowska 40 (1986), 251-265.

[15] G. N. Watson, A Treatise on the Theory of Bessel Functions, Second edition, Cambridge University Press, Cambridge, London and New York, 1944.

[16] N. Yağmur and H. Orhan, Starlikeness and convexity of generalized Struve functions, Abstr. Appl. Anal. 2013 (2013), article ID 954513, 6 pages; doi:10.1155/2013/954513.

[17] — Hardy space of generalized Struve functions, Complex Var. Elliptic Equ. 59 (2014), no. 7, 929-936.

Department of Mathematics FACUlTy of SCIENCE AND ART

ERZINCAN UNIVERSITY

ERZINCAN, 24000, TURKEY

E-mail address: nhtyagmur@gmail.com 